\title{
Research on the regeneration technology of etching waste solution
}

\author{
Chen $\mathrm{Ru}^{*}$ \\ College of Information Engineering, Shaanxi Institute of International Trade \& Commerce, Xi'an 712046, Shaanxi, China
}

\begin{abstract}
With the increase in the use of electronic products, the consumption of circuit boards also increased sharply, the amount of waste liquid discharged in the process of producing circuit boards will follow the increase, and these waste liquids, if discharged directly, not only cause a great waste of resources, but also cause serious water pollution, soil pollution, and thus with the food chain into the body of people and animals, seriously endangering human health, so we need to Therefore, we need to recycle and utilize this resource. In this paper, we adopt the recycling treatment system to transport the waste solution from the production line to the electrolyzer, and the overflow tank stores the electrolyte discharged from the production line. We adopt the waste electrolyte after treating the micro-etching waste solution for waste solution recycling treatment, and electrolyze the micro-etching waste solution again after treatment, and use it repeatedly to improve the utilization rate of electrolyte.
\end{abstract}

\section{Introduction}

PCB (printed circuit board) in the electronic information industry chain plays a vital role in the top and bottom, that is, integrated circuits can not be separated from the printed circuit board, military and civilian, high-tech electronic products can not do without the printed circuit board [1], in the next few years, in the automotive, smart grid, medical equipment, aerospace and military and other applications to increase the circuit board. Printed circuit board is the key electronic interconnections of electronic products, "the mother of electronic products," said.

With the increase in demand for electronic products, the need for PCB circuit boards is also increasing year by year. Printed circuit board industry upstream raw materials including copper laminates, copper balls, copper foil, semi-curing sheet, gold salt, ink and dry film; PCB in the downstream field of application of the breadth of electronic products irreplaceable and the need to decide. For the current application areas, the global field of largescale application of PCB mainly in the field of computers, communications, consumer electronics, in these three areas of application scale accounted for $70 \%$ of the overall PCB application scale [2]. The PCB industry chain is shown in the following figure:



Figure 1 PCB industry chain

The laminate industry is an industry with a large capital requirement and relatively high concentration.In 2015, the global total output value of rigid copper laminates (including semi-curable sheets) was $\$ 9,299$ million, and the total output value in Asia was $\$ 8,699$ million, including \$6,043 million in mainland China, \$509 million in Japan, and \$2,217 million in other Asian countries. Similar to the PCB industry, the production of copper laminates is mainly concentrated in the Asian region, the global rigid copper laminates in 2015 by production statistics of 517.5 million square meters, Asia 502 million square meters, accounting for $97.00 \%$ of the world, including 361.9 million square meters in mainland China, China Taiwan 56.2 million square meters, South Korea 32.3 million square meters, Japan 0.192 million square meters. According to the China Printed Circuit Association (CPCA) report, China's printed circuit board manufacturers have about 1,000 about the total output value of about 100 billion yuan, has surpassed Japan to rank first in the world. The development of China's PCB upstream industry is mature, well supported and well supplied, providing a good environment for the development of the PCB industry[5].

\footnotetext{
*Corresponding author: 24131266@qq.com
} 
The production of PCB is a leading industry in our province, with the expanding scale of industrial industries in our province and the increasing popularity of printed board production, the number of electronic equipment factories is increasing[6]. The treatment of waste liquids is an important part of the production that must be handled. The random discharge of waste liquid will cause a great waste of resources, so this wasted resource can be recycled and reused. And if the waste liquid is discharged arbitrarily without treatment, it will cause serious water pollution and soil pollution, and thus enter the body of human and animals with the food chain, seriously endangering human health.

PCB production to consume a large number of copper clad board, the annual consumption of copper accounted for $30 \%$ of the total national consumption, and in the production process of etching, micro-etching, more than $50 \%$ to $60 \%$ of its substrate copper clad board to be etched into waste etching solution, resulting in a large amount of high concentration of copper wastewater, waste liquid out, seriously polluting the environment [3], a great danger to human health, The amount of copper in the waste stream is shown in Figure 2.

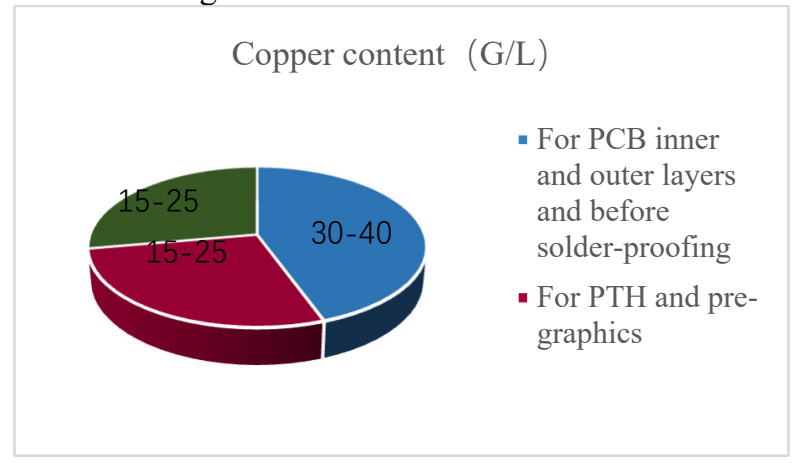

Figure 2 Copper content in waste liquid

\section{Technical route}

Combined with the actual production of the enterprise, the waste liquid generated in the production line is firstly recycled to the overflow tank, and this equipment adopts non-toxic, sanitary, high temperature resistant and recyclable PP pipe. Then the waste liquid in the overflow tank is transferred to the storage barrel, because when the waste liquid is processed, the waste liquid has to be transferred to the electrolytic tank continuously, and this transfer process is extracted by pump, so the waste liquid in the production line flows to the overflow tank first, and then the waste liquid in the overflow tank is extracted to the storage barrel by pump (to prevent the overflow tank from being empty when there is no waste liquid in the production line, which burns out the pump and stores the waste liquid in advance). If the liquid level reaches the highest point, the pump between the overflow tank and the storage tank will stop working; if the liquid level reaches the lowest point, the pump between the storage tank and the electrolysis tank will stop working. The waste liquid in the storage barrel is extracted to the electrolytic tank through the pump. The anode plate used in the electrolysis of the electrolytic tank is crucial, and titanium with good corrosion resistance, stable chemical properties, good resistance to high temperature, low temperature, strong acid and strong alkali is used in this project, and the surface coating of the titanium plate is a precious metal of iridium-tantalum alloy as the anode, and this plate is relatively expensive but stable in performance. Cathode using inexpensive stainless steel plate, electrolysis will be copper plated on the stainless steel plate, a few days later it can be torn off to form a copper foil.

When the electrolyte is not enough, the electrolyte can be directly extracted from the inside of the storage barrel, which can prevent the pump from being damaged due to the electrolyte being pumped out. After treatment, the electrolysis of micro-etching waste solution is carried out again, which can be used repeatedly to improve the utilization rate of electrolyte, and the fan is used to ventilate the inside of the electrolysis tank, and the copper is taken off after drying quickly. The hydrogen is discharged to the standard, and the electrolyte is circulated in the whole device to minimize the contact with the outside environment and reduce pollution. The schematic diagram of the system structure is shown in Figure 3.



Figure 3 The schematic diagram of the system structure Among them, 1 - Production line discharge pipe, 2 Overflow tank, 3 - Infrared level detector, 4 - Pipeline, 5-Pump, 6-Storage drum, 7-Low level detector, 8-High level detector,

9-Pump,10-Electrolysis tank, 11-Concentration monitoring probe, 12-Pump,

13-Mixer, 14-Solenoid valve,15-Sealing cover, 16-Fan, 17-Waste liquid mediation box

Figure 3 System structure schematic

(1) Working principle.

The waste liquid from the production line is injected into the overflow tank 2 through the discharge pipe 1 of the production line for centralized collection, and when the overflow tank reaches a certain liquid level, the infrared liquid level detector 3 transmits the signal to the control box to automatically start the pump 5 to inject the waste liquid into the storage barrel 6 , and the amount of waste liquid in the storage barrel 6 is detected by the infrared liquid level detector 7 and infrared liquid level detector 8 to prevent the pump 9 from dry pumping when the waste liquid in the storage barrel 6 is insufficient. The waste liquid flows into the electrolytic tank 10 for electrolysis driven by pump 9 . When the liquid level of the storage barrel is not enough to pump the whole electrolytic tank, pump 9 is automatically turned off, and then the infrared level detector checks whether there is waste liquid in the overflow tank 1, and if the liquid level is checked, then pump 5 is automatically turned on, and after injecting a 
certain amount pump 9 is automatically turned on to continue to inject waste liquid into the electrolytic tank to make it reach a full tank of waste liquid, and then turn on electrolysis, the concentration of electrolyte is detected by concentration detection probe 11 , and the electrolyte is circulated in electrolytic tank 10 by pump 12, and when chemical reaction occurs (chemical equation is as follows:) The reaction of the cathode is:

$$
\mathrm{Cu}^{2+}+2 e \rightarrow \mathrm{Cu} \mathrm{Cu}^{+}+e \rightarrow C u
$$

The reaction of the anode is:

$$
2 \mathrm{OH}^{-}-2 e \rightarrow \mathrm{O}_{2} \uparrow+\mathrm{H}_{2} \uparrow
$$

The copper ions around the titanium plate are reduced, so the concentration of the electrolyte in the electrolytic cell 10 is made the same by using the mixer 13 to stir.

After the electrolysis is completed, the solenoid valve 14 opens to flow the waste liquid after electrolysis into the waste liquid mediation box and then discharges it. After all the electrolyte inside the electrolytic tank 10 flows out, the fan 16 blows wind to the inside of the electrolytic tank 10 to dry the copper monolith quickly, and after the copper monolith is blown dry, the sealed cover 15 is opened to take out the copper monolith.

(2) Electrolysis tank: tank, liquid supply tank, electrolysis power supply and liquid discharge tank, the upper part of the tank is provided with liquid supply pipe, the tank is connected to the liquid supply tank through the liquid supply pipe, the bottom of the tank is provided with liquid discharge pipe, the tank is connected to the liquid discharge tank through the liquid discharge pipe, the liquid discharge tank is connected to the electrolysis treatment tank in the middle of the liquid supply tank and connected to the tank through the pipe, the tank includes a shell, a heating pipe, a steel plate and a titanium plate, the heating pipe is evenly distributed in the interior of the shell, and The heating tubes are evenly distributed inside the shell, and the two ends of the heating tubes are connected to the heat source, the titanium plate and the steel plate are vertically located inside the tank, and the titanium plate is connected to the anode of the electrolysis power supply, and the steel plate is connected to the cathode of the electrolysis power supply, and the electrolysis power supply is located on the top of the tank. The diagram of the electrolysis tank is shown in Figure 4 below.



Figure 4 Electrolysis tank

Where: 1-Tank, 2-Supply tank, 3-Discharge tank, 4Supply pipe, 5-Discharge pipe, 6-Electrolytic treatment tank, 7-Pipe, 8-Heating pipe, 9-Electrolyte, 10-Titanium plate, 11-Steel plate, 12-Exhaust pipe, 12-Electrolyte density sensor, 13-Valve, 14-Power supply, 15-Anode, 16-Cathode.

The upper part of the tank 1 is equipped with a liquid supply pipe 4 , and the tank 1 is connected to the liquid supply tank 2 through the liquid supply pipe 4 , the bottom of the tank 1 is equipped with a liquid discharge pipe 5, and the tank 1 is connected to the liquid discharge tank 3 through the liquid discharge pipe 5, and the middle of the liquid discharge tank 3 and the liquid supply tank 2 is equipped with an electrolytic treatment tank 6 , which is connected to each other through the pipe 7 . At the same time, the electrolytic treatment tank 6 is used to treat the discharged electrolyte 9 , which flows into the inlet tank after treatment, so that the waste liquid can be recycled, and the electrolyte density sensor 12 is used to intelligently control the opening and closing of the valve 13, which ensures the density of the electrolyte 9 and achieves an intelligent effect, and at the same time plays a role in reducing costs and increasing resource utilization. It can effectively improve the electrolytic capacity inside the tank, increase the copper precipitation rate and copper grade, while using the electrolytic treatment tank to treat the discharged electrolyte and flow into the inlet tank after treatment, so that the waste liquid can be recycled, and using the electrolyte density sensor to intelligently control the opening and closing of the valve to ensure the density of the electrolyte and achieve the intelligent In order to avoid vaporization of electrolyte, a sealed shell is used to ensure that the air is not polluted and to protect the environment. The titanium plate is used as the anode, which is not easy to be consumed, and the purity of the electrolytic copper is very high, basically reaching over $99.95 \%$ purity.

(3) Goals Achieved

* The purity of copper obtained by this method can reach $99.95 \%$ to $99.98 \%$, meeting the requirements of national standards.

* The total copper removal rate of the discharged wastewater is $>98 \%$, without any pollution to the environment.

* The microetching solution after electrolysis can also continue to be recycled by adding an appropriate amount of sulfuric acid, achieving zero emission.

This method is fast, low energy consumption, and the whole process is completed by fully automatic mechanical control, with little human intervention, saving human resources costs; achieving the recycling of resources; and achieving environmental protection.

\section{Difficulties of the technology.}

Using this technology is mainly in the control of current during operation, if the current is too high, it will cause the coating of anode plate to fall off to reduce its service life, and the copper electrolyzed is not dense and smooth; with the growth of energizing time, the cation (copper ion) of sulfuric acid solution is extremely reduced, the energizing performance is getting worse and the current is getting weaker, so the control of current size is crucial in this project. 


\section{Conclusion}

The method is used to treat the waste liquid produced by the production of circuit boards, and the appropriate amount of sulfuric acid is added to the etching solution after electrolysis can also continue to be recycled to achieve zero emissions, and the purity of copper obtained using this technology can reach $99.95 \%$ to 99.98 , which meets national standards, and the total copper removal rate of the discharged wastewater is greater than $98 \%$, with no pollution to the environment. Of course, there are still shortcomings in this method, which is also the direction of future research needed.

\section{Acknowledgements}

This paper is supported by Xi'an Science and technology plan project (2020KJRC0150); Key R \& D plan project of Shaanxi Province (2019GY-038); Shaanxi Province Natural Science Basic Research Program (2021JM-539); Scientific and technological innovation team of computer vision and image processing technology.

\section{References}

1. Chen Ru. The impact of the development of printed circuit board industry on China's economy [J]. Journal of Economic Research,2017(28):38-39.

2. Chen $\mathrm{Ru}$. The impact of the demand for electronic products on PCB $[\mathrm{J}]$. Journal of Economic Research,2017(33):29+38.

3. Wang Hui. Separation and recovery of copper from copper-containing etchants - separation of copper sulfate and sodium sulfate and sulfuric acid [C]// Proceedings of the 4th National Symposium on Application of Membrane Separation Technology in Metallurgical Industry. ,2014:230-231.

4. Liu Houchuan,Qi Jianjian,Lu Zhaohui,Tang Zhengtao, Yu Shaoming. Research progress of printed circuit board etching and copper-containing etching waste liquid treatment technology[J]. Contemporary Chemical Research,2019(15):109110.

5. Xu Qiang,Li Chen,Du Jun. Technical solutions and engineering applications of wastewater treatment for printed circuit board production[J]. Resource Conservation and Environmental Protection,2019(03):102-103+108.

6. Peng Yiming. Research and discussion on the treatment and recycling of circuit board browning waste liquid[J]. Resource Conservation and Environmental Protection,2020(01):94. 